\section{PLEASANT HANDLING; EASY CLEAN-UP}

GC Fuji TEMP LT is specially designed for longterm temporary cementation. It is very convenient during application and provides a stable retention while assuring the future safe removal of indirect restorations.

GC Fuji TEMP LT has everything you need in temporary cement: pleasant handling; no runny consistency with low film thickness; easy to remove excess; reliable retention; long term security; no negative influence on final adhesive cementation; secure retrievability; safe removal of the restoration whenever needed; and easy clean-up.

These advantages combined with the safety offered by glass ionomers make it the ideal choice for long-term temporary cementation of all types of all-ceramic, resin, acrylic and metalbased crowns and bridges, including try-on cementation of long-span prosthetic appliances. It is also specially adapted to assure sufficient retention and retrievability of crowns and bridges cemented on implant abutments.

GC Fuji TEMP LT comes in one single shade (Universal) and is presented in paste pack cartridges to be used with the practical Paste Pack Dispenser from GC. Removal of cemented prosthetic appliances is better performed with the use of GC Pliers for better control of applied forces.

For further information contact GC UK on 01908218999 or email info@uk.gceurope.com.

\section{IMPRESSIVE LONG-TERM IMPLANT OUTCOMES}

One of the largest long-term clinical studies on the survival and success of dental implants has been conducted at the University of Bern in Switzerland. The study assessed the outcome of 511 Straumann SLA tissue-level implants in 303 patients over ten years.

Its authors concluded that their retrospective analysis resulted in a ten-year implant survival rate of $98.8 \%$ and a success rate of $97.0 \%$. In addition, the prevalence of peri-implantitis in a large cohort of orally healthy patients was low with $1.8 \%$ during the ten-year period. No implant fracture was noted.

This study is one of the first large ten-year clinical studies to document a dental implant of this kind that is still on the market. The Straumann SLA tissue-level implant is one of the most extensively studied dental implants and these long-term results explain why it is also one of the most widely used.

Visit http://onlinelibrary.wiley.com/doi/10.1111/ j.1708-8208.2012.00456.x/full for details and see www.straumann.com.

\title{
HIGHEST LEVEL OF PREDICTABILITY EVER
}

The Piezotome 2, from dental technology specialists Acteon, enables you to carry out implant dentistry with the highest level of predictability ever possible.

This piezoelectric ultrasonic generator for pre-implant and periodontal surgery makes hard tissue surgery much more precise and significantly less traumatic to both hard and soft tissues. This in turn means dramatically less post-operative discomfort and swelling for your patients.

The Piezotome unit allows successful negotiation of complex septae, providing the patient with fewer surgical interventions and subsequently a shorter treatment duration. It has further use in ridge splitting and expansion with success even in the mandible. The Piezotome's conservative tips minimise bone removal when preparing the osteotomy coupled with an increased directional accuracy.

The Piezotome 2 comes with specifically designed extraction tips. This unit has certain advantages over others, as it has a fibre optic light which allows clear vision into the periodontal ligament space and surrounding tissues. It also has an extremely wide range of power settings which allows it to adapt to the clinical situation by providing either gentle luxation, or a more vigorous manipulation quickly and easily.

Call 01480477307 or email: info@acteongroup.co.uk to arrange an on-site demo at a suitable time for you.

\section{PROBLEM FREE COMPRESSED AIR}

Dental Air Services, the leading supplier of oil-free compressed air packages within the dental industry, would like to introduce their 'Air Care Adoption Programme'.

For a fixed monthly cost Dental Air Services will adopt your existing compressor on to their service and support package. Following the first service visit all necessary documentation will be issued to satisfy the HSE legal requirements for regular servicing. Dental Air have extended their service support packages to all makes of existing compressors.

Air qualities will also be tested during the servicing and, if appropriate, certification will be issued to comply with NHS HTM 2022/1 which will also satisfy any CQC inspection concerns.

For further details of the service support packages call 08009757530 for an instant quotation; emailinfo@ dentalair.com or visit www. dentalair.com. 